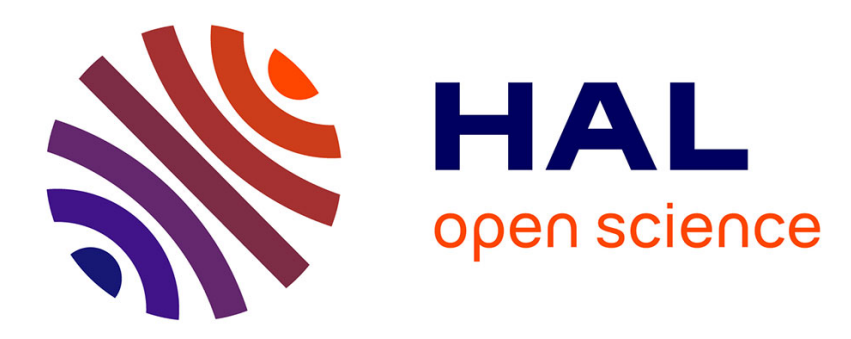

\title{
First passage times of subordinators and urns Philippe Marchal
}

\section{- To cite this version:}

Philippe Marchal. First passage times of subordinators and urns. A lifetime of excursions through random walks and Lévy processes, 2021. hal-03063774

\section{HAL Id: hal-03063774 https://hal.science/hal-03063774}

Submitted on 14 Dec 2020

HAL is a multi-disciplinary open access archive for the deposit and dissemination of scientific research documents, whether they are published or not. The documents may come from teaching and research institutions in France or abroad, or from public or private research centers.
L'archive ouverte pluridisciplinaire HAL, est destinée au dépôt et à la diffusion de documents scientifiques de niveau recherche, publiés ou non, émanant des établissements d'enseignement et de recherche français ou étrangers, des laboratoires publics ou privés. 


\title{
First passage times of subordinators and urns
}

\author{
P. Marchal \\ Université Paris Sorbonne Nord \\ -LAGA \\ CNRS (UMR 7539) \\ F-93430, Villetaneuse \\ marchal@math.univ-paris13.fr
}

\begin{abstract}
It is well-known that the first time a stable subordinator reaches $[1,+\infty)$. is MittagLeffler distributed. These distributions also appear as limiting distributions in triangular Polya urns. We give a direct link between these two results, using a previous construction of the range of stable subordinators. Beyond the stable case, we show that for a subclass of complete subordinators in the domain of attraction of stable subordinators, the law of the first passage time is given by the limit of an urn with the same replacement rule but with a random initial composition.
\end{abstract}

Keywords: stable subordinator, first passage time, Polya urn

\section{Introduction}

Let $\left(S_{t}\right)_{t \geq 0}$ be a stable subordinator of index $\alpha \in(0,1)$, started at 0 , and let $T$ be the first passage time in $[1,+\infty)$.

$$
T=\inf \left\{t>0, S_{t}>1\right\}
$$

Then it is well-known that the law of $T$ is the Mittag-Leffler distribution with parameter $\alpha$, which is characterized by its moments:

$$
\mathbb{E} T^{n}=\frac{\Gamma(1 / \alpha+n)}{\Gamma(1 / \alpha) \Gamma(1+n \alpha)}
$$

See for instance [10], p.10. This same distribution also appears as the asymptotic number of white balls in a classical Polya urn scheme. Let us introduce some standard notation.

\section{Definition}

We call an urn scheme with replacement matrix

$$
\left(\begin{array}{ll}
a & b \\
c & d
\end{array}\right)
$$


and initial condition $\left(B_{0}, W_{0}\right)$ the following process. We initially have a black and a white ball with respective weights $\left(B_{0}, W_{0}\right)$. Then sequentially, a ball is drawn at random with probability proportional to its weight. If this ball is black, it is replaced into the urn together with a black ball of weight $a$ and a white ball of weight $b$. If the ball is white, it is put back into the urn together with a black ball with weight $c$ and a black ball with weight $d$.

Consider the case with the replacement matrix

$$
\left(\begin{array}{cc}
1 & 0 \\
1-\alpha & \alpha
\end{array}\right)
$$

and initial condition $\left(B_{0}, W_{0}\right)$. Let $W_{n}$ be the total weight of white balls after $n$ steps. Then $n^{-\alpha} W_{n}$ converges in law to a Mittag-Leffler random variable $X$ which can be characterized by its moments, namely

$$
\mathbb{E} X^{n}=\frac{\Gamma\left(B_{0}+W_{0}\right) \Gamma\left(W_{0} / \alpha+n\right)}{\Gamma\left(W_{0} / \alpha\right) \Gamma\left(B_{0}+W_{0}+n \alpha\right)}
$$

See e.g. [6]. In particular, $X$ has the same law as the first passage time $T$ defined above with the choice of parameters $\left(B_{0}, W_{0}\right)=(1-\alpha, \alpha)$. Note that $(2)$ still holds when $\alpha=1$.

We argue that these two results are directly related via a construction of stable subordinators that first appeared in [7] and that was then extended to complete subordinators in [8]. Complete subordinators can be indexed by all possible measurable functions $\beta:[0,1] \rightarrow[0,1]$ and have Lévy-Khintchine exponent given by

$$
\phi^{(\beta)}(\lambda)=-\log \mathbb{E}\left[\exp \left(-\lambda S_{1}^{(\beta)}\right)\right]=\exp \int_{0}^{1} \frac{(\lambda-1) \beta(x)}{1+(\lambda-1) x} d x
$$

For general references on subordinators, see e.g. [2] and [11]. Our result is that for a subclass of complete subordinators, the first passage time is also related to an urn process:

Theorem 1 Let $\beta:[0,1] \rightarrow[0,1]$ be a measurable function which is constant, equal to $\alpha \in(0,1]$ on an interval $[0, h]$ for some $h \in(0,1]$. Let $\left(S_{t}^{(\beta)}\right)$ be the subordinator with exponent given by (3) and let

$$
T^{(\beta)}=\inf \left\{t>0, S_{t}^{(\beta)}>1\right\}
$$

be its first passage time to $[1,+\infty)$. Then, up to a multiplicative constant, $T^{(\beta)}$ has the same law as the limit of $n^{-\alpha} W_{n}$ where $W_{n}$ is the total weight of white balls in an urn scheme with replacement matrix (1) and random initial conditions as follows. Put $\theta=(1 / h)-1$. Then for all integers $l, m \geq 0$,

$$
\mathbb{P}\left(\left(B_{0}, W_{0}\right)=(l+(m+1)(1-\alpha),(m+1) \alpha)=\frac{\theta^{l} e^{-\theta}}{l !} \frac{1}{2 i \pi} \int_{\mathcal{C}} \frac{d t}{t} \psi(t)^{m}(1-\psi(t)) \frac{1-(1 / t)^{l+1}}{1-(1 / t)}\right.
$$

where $\mathcal{C}$ is the unit circle of the complex plane and the function $\psi$ is given by

$$
\psi(t)=1-\exp \left(\int_{0}^{1} \frac{t \gamma(x)}{1-t x} d t\right)
$$

with

$$
\gamma(x)=\beta\left(\frac{1}{\theta+1-\theta x}\right)
$$


The presence of a multiplicative constant in Theorem 1 is not a real issue since this corresponds to replacing $\left(S_{t}^{(\beta)}\right)$ with $\left(S_{c t}^{(\beta)}\right)$ for some positive constant $c$. We stated our result for the entrance to $[1,+\infty)$ but of course, similar results hold with a staightforward adaptation for the entrance to $[a,+\infty)$ for any $a>0$.

When $h=1$, the subordinator is stable. When $h<1$, the process is in the domain of attraction of an $\alpha$-stable subordinator in small time: as $t \rightarrow 0, t^{-1 / \alpha} S_{t}^{(\beta)}$ converges in law to the (unique) positive stable distribution with index $\alpha$.

Conversely however, a complete subordinator $\left(S_{t}^{(\beta)}\right)$ may belong to the domain of attraction of an $\alpha$-stable subordinator in small time without the function $\beta$ being constant near 0 . Take for instance $\beta(x)=\alpha+(1-\alpha) x$, then $\left(S_{t}^{(\beta)}\right)$ belongs to this domain of attraction but Theorem 1 does not apply. It would be interesting to know how far Theorem 1 could be generalized for subordinators of this kind, that is, whether the first passage time can be related to an urn process.

Note that if the hypothesis of Theorem 1 on $\beta$ is satisfied with $\alpha=1$, the subordinator has positive drift whereas if it is satisfied with $\alpha=0$, the subordinator is a compound Poisson process, see [8]. In the case $\alpha=1$, Theorem 1 still holds. On the other hand, in the case $\alpha=0$, the first passage time problem reduces to a problem on random walks which can be handled using the same tools as in Section 3.3. This last case is in fact very classical and we shall not review the corresponding literature here.

Using Theorem 1 and (2), one can compute the moments of the first passage time. Let us make these computations in two simple cases. First, suppose that

$$
\beta(x)=\alpha \mathbf{1}_{\{x \in[0, h]}
$$

Then almost surely, $W_{0}=\alpha$ and the the moments of $T$ are given by (with $c$ a positive constant and $\theta$ as in Theorem 1):

$$
\mathbb{E} T^{n}=c^{n} \sum_{l \geq 0} \frac{\theta^{l} e^{-\theta}}{l !} \frac{\Gamma(1+n)}{\Gamma(1+l+n \alpha)}
$$

Next, suppose that

$$
\beta(x)=\alpha \mathbf{1}_{\{x \in[0, h)\}}+\mathbf{1}_{\{x \in[h, 1]\}}
$$

Then almost surely, $B_{0}=1-\alpha$ and the the moments of $T$ are given by:

$$
\mathbb{E} T^{n}=c^{n} \sum_{m \geq 0} \frac{\theta^{m} e^{-\theta}}{m !} \frac{\Gamma(1+m+n)}{\Gamma(1+m+n \alpha)}
$$

In all other cases however, the computations are more intricate and there are no obvious simplifications.

Apart from exact computations, for which little is known, other results on first passage times for subordinators, regarding in particular the existence of a density or asymptotic estimates, can be found in [5] and references therein.

The remainder of this paper is organized as follows. We first recall the construction of regenerative sets from [8], both in the discrete and continuous case, in Section 2. We explain in Section 3 how urns are embedded in this construction and how the distributions described in Theorem 1 occur in that context. Finally, we show in Section 4 that the embedded urns described in Section 3 indeed correspond to first passage times for subordinators. 
Figure 1: Construction 1

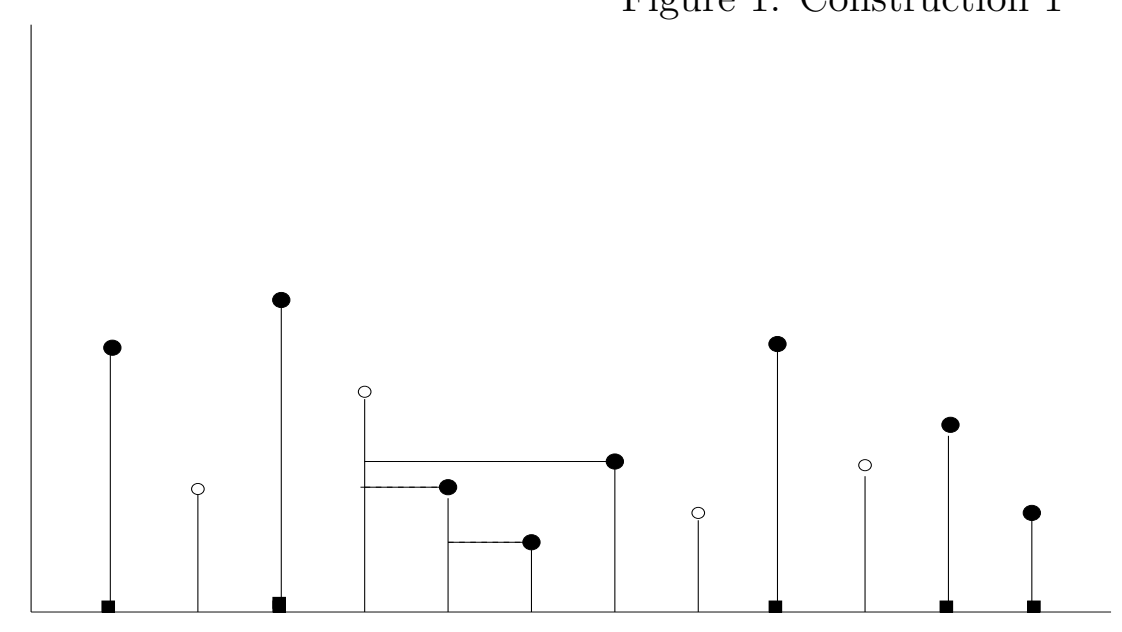

\section{A construction of regenerative sets}

In the first two subsections, we recall the construction of regenerative sets given in [8], both in the discrete and in the continuous case. The proof of Theorems 2 and 3 can be found there. The class of regenerative sets obtained in Section 2.2 is exactly the class of ranges of complete subordinators, as noted in [1] and [4].

\subsection{The lattice case}

We begin by the construction of regenerative sets in $\mathbb{N}$.

\section{Construction 1.}

Fix a measurable function $\gamma:[0,1] \rightarrow[0,1]$. Let $\left(X_{n}, n \geq 1\right)$ be iid random variables, uniformly distributed on $[0,1]^{2}$. We denote $X_{n}=\left(h_{n}, U_{n}\right)$. One should view $h$ as a height and $U$ as a parameter. Say that $X_{n}$ is green if $U_{n} \leq \gamma\left(h_{n}\right)$, and red otherwise. Say that an integer $k \in[1, n]$ is $n$-visible if $h_{k} \geq h_{m}$ for all integers $m \in[k, n]$. Finally, say that $n$ percolates for $\gamma$ if, for every $k \leq n$ such that $k$ is $n$-visible, $X_{k}$ is green. Let $R^{(\gamma)}$ be the set of integers that percolate for $\gamma$ (by convention, 0 percolates for $\gamma$ ).

See Figure 1. Green points are represented by black circles, red points by white circles and the black squares stand for the integers that percolate. The horizontal lines express the fact that the red point at 4 prevents 5, 6 and 7 from percolating.

Remark that if $\gamma$ is a constant, then the $X_{n}$ are green or red with probability $\gamma$ (resp. $1-\gamma)$, independently of the height.

Theorem 2 The set $R^{(\gamma)}$ defined by Construction 1 is a lattice regenerative set. It can be viewed as the image of a random walk $\left(S_{n}^{(\gamma)}, n \geq 0\right)$, where $S_{n}^{(\gamma)}=Y_{1}^{(\gamma)}+\ldots+Y_{n}^{(\gamma)}$, the $Y_{i}^{(\gamma)}$ being iid random variables taking values in $\mathbb{N} \cup\{\infty\}$, with generating function

$$
\psi^{(\gamma)}(t)=\mathbb{E}\left(t^{Y_{1}^{(\gamma)}}\right)=1-\exp \left(-\int_{0}^{1} \frac{t \gamma(x)}{1-t x} d x\right)
$$




\subsection{The continuous case}

Consider a Poisson Point process $\mathcal{N}$ on $\mathbb{R}_{+} \times[0,1] \times[0,1]$ with intensity $d x \otimes y^{-2} d y \otimes d z$. Given a measurable function $\beta:[0,1] \rightarrow[0,1]$, we can define an analogue of Construction 1 as follows.

\section{Construction 2.}

Say that a point $X=(t, h, U)$ of $\mathcal{N}$ is green if $U \leq \beta(h)$, and red otherwise. Say that another point $X^{\prime}=\left(t^{\prime}, h^{\prime}, U^{\prime}\right)$ of $\mathcal{N}$ is visible for $X$ if $t^{\prime} \leq t$ and if, for all points of $\mathcal{N}$ of the form $X^{\prime \prime}=\left(t^{\prime \prime}, h^{\prime \prime}, u^{\prime \prime}\right)$ with $t^{\prime} \leq t^{\prime \prime} \leq t$, we have $h^{\prime} \geq h^{\prime \prime}$. Finally, say that $X$ percolates for $\beta$ if, for every $X^{\prime}$ such that $X^{\prime}$ is visible for $X, X^{\prime}$ is green. By convention, 0 percolates for $\beta$. We denote by $\mathcal{R}_{1}^{(\beta)}$ the set of first coordinates of percolating points, and we set

$$
\mathcal{R}^{(\beta)}=\overline{\mathcal{R}_{1}^{(\beta)}}
$$

For every point $X=(t, h, U)$ of $\mathcal{N}$, let $U(X)$ be the set of points of $\mathcal{N}$ of the form $X^{\prime}=\left(t^{\prime}, h^{\prime}, u^{\prime}\right)$ with $t^{\prime} \leq t$ and $h^{\prime} \geq h$. Then almost surely, $U(X)$ is finite, since almost surely, every strip of the form $[0, t] \times[h, \infty] \times[0,1]$ with $h>0$ contains a finite number of points of $\mathcal{N}$. Moreover, determining whether $X$ percolates only depends on $U(X)$, and therefore Construction 2 is well-defined.

Theorem 3 The set $\mathcal{R}^{(\beta)}$ defined by Construction 2 is a regenerative set. It can be viewed as the image of a subordinator $\left(S_{t}^{(\beta)}\right)_{t \geq 0}$ with Laplace exponent

$$
\phi^{(\beta)}(\lambda)=-\log \mathbb{E}\left[\exp \left(-\lambda S_{1}^{(\beta)}\right)\right]=\exp \int_{0}^{1} \frac{(\lambda-1) \beta(x)}{1+(\lambda-1) x} d x
$$

for $\lambda \geq 0$.

\subsection{Relating the discrete and the continuous case}

Let $h>0$. As noted above, if we only look at the points of $\mathcal{N}$ with $y$-coordinate $\geq h$ in Construction 2, we have a discrete set and we can determine whether these points percolate or not without taking into account the points whose $y$-coordinate is $<h$. Denote the points with $y$-coordinate $\geq h$ by

$$
\left(x_{1}, y_{1}, U_{1}\right),\left(x_{2}, y_{2}, U_{2}\right), \ldots
$$

with $x_{1}<x_{2}<\ldots$. From this discrete set, we can recover Construction 1 as follows.

Let $\theta=(1 / h)-1$ and consider the function $F:[h, 1] \rightarrow[0,1]$ defined by

$$
F(x)=1+\frac{1}{\theta}-\frac{1}{\theta x}
$$

Its inverse is the function $F^{-1}:[0,1] \rightarrow[h, 1]$ given by

$$
F^{-1}(x)=\frac{1}{\theta+1-\theta x}
$$


Put $h_{n}=F\left(y_{n}\right)$ for every $n \geq 1$. Then it is easily seen that the sequence $\left(h_{n}, n \geq 1\right)$ is a sequence of iid random variables, uniformly distributed on $[0,1]$ and independent of the sequence $\left(x_{n}, n \geq 1\right)$. Therefore the sequence

$$
\left(\left(h_{n}, U_{n}\right), n \geq 1\right)
$$

has the same law as in Section 2.1 and is independent of $\left(x_{n}, n \geq 1\right)$.

Consider the function

$$
\gamma(x)=\beta\left(F^{-1}(x)\right)=\beta\left(\frac{1}{\theta+1-\theta x}\right)
$$

so that $\beta(x)=\gamma(F(x))$. Then from the sequence $\left(h_{n}, U_{n}\right)$ and the function $\gamma$, we can define a regenerative set $R$ by Construction 1 and we check that $k \in R$ if and only if $\left(x_{k}, y_{k}, U_{k}\right)$ percolates by Construction 2. Moreover, Theorem 2 tells us that $R$ is the range of a random walk $\left(S_{n}\right)$ with generating function

$$
\psi(t)=1-\exp \left(\int_{0}^{1} \frac{t \gamma(x)}{1-t x}\right)
$$

which is the same as in Theorem 1.

\section{$3 \quad$ Embedded urns}

In this section, we use the construction of regenerative sets from Section 2.2. We shall always restrict ourselves to the subset of points of $\mathcal{N}$ with $x$-coordinate $\leq 1$.

\subsection{An alternative description of the urn}

Consider an urn scheme with replacement matrix

$$
\left(\begin{array}{cc}
1 & 0 \\
1-\alpha & \alpha
\end{array}\right)
$$

and initial condition $\left(B_{0}, W_{0}\right)$. This urn can be described by the following mechanism:

- At time 0 , add a black ball with weight $B_{0}$ and a white ball with weight $W_{0}$.

- Recursively at time $N \geq 1$,

- choose, independently of the past, a random time $t_{N} \in\{0, \ldots N-1\}$ with probability

$$
\mathbb{P}\left(t_{N}=k\right)=\frac{1}{N-1+B_{0}+W_{0}}
$$

if $k \geq 1$ and

$$
\mathbb{P}\left(t_{N}=0\right)=\frac{B_{0}+W_{0}}{N-1+B_{0}+W_{0}}
$$


- If $t_{N}=0$, then at time $N$, then with probability $B_{0} /\left(B_{0}+W_{0}\right)$, add a black ball with weight 1 and with probability $W_{0} /\left(B_{0}+W_{0}\right)$, add a black ball with weight $1-\alpha$ and a white ball with weight $\alpha$.

- If, at time $t_{N} \geq 1$, a black ball with weight 1 had been added, then add at time $N$ a black ball with weight 1 .

- If, at time $t_{N} \geq 1$, a black ball with weight $1-\alpha$ and a white ball with weight $\alpha$ had been added, then at time $N$, with probability $1-\alpha$, add a black ball with weight 1 and with probability $\alpha$, add a black ball with weight $1-\alpha$ and a white ball with weight $\alpha$.

\subsection{The stable case}

We deal here with the case when $\beta$ is constant and equal to $\alpha \in(0,1)$. Let us denote the set of points of $\mathcal{N}$, re-arranged by decreasing $y$-coordinate, as

$$
\left\{\left(x_{1}, y_{1}, U_{1}\right),\left(x_{2}, y_{2}, U_{2}\right), \ldots\right\}
$$

with $y_{1}>y_{2} \ldots$ By convention, set $\left(x_{0}, y_{0}\right)=(0, \infty)$.

For two integers $N \geq 0$ and $k \in[0, N]$, put

$$
z_{k}^{(N)}=\min \left(\left\{x \in\left\{1, x_{0}, \ldots, x_{N}\right\}, x>x_{k}\right\}\right)
$$

and

$$
I_{k}^{(N)}=\left(x_{k}, z_{k}^{(N)}\right]
$$

In words, $x_{0}, \ldots, x_{N}$ cut the interval $[0,1]$ into $N+1$ subintervals and $I_{k}^{(N)}$ is the subinterval with left extremity $x_{k}$. Denote the lengths of these subintervals

$$
l_{k}^{(N)}=z_{k}^{(N)}-x_{k}
$$

Let $Q_{N+1}$ be the index of the interval where $x_{N+1}$ lies, that is, put $Q_{N+1}=k$ if $k$ is the (unique) integer $\in[0, N]$ such that $x_{N+1} \in I_{k}^{(N)}$. From the properties of Poisson point processes, the random variable $\left(l_{0}^{(N)}, \ldots, l_{N}^{(N)}\right)$ is uniformly distributed on the $N$-dimensional simplex and is independent of the random variables $Q_{i}, 1 \leq i \leq N$. Therefore, for every $k \in[0, N]$,

$$
\mathbb{P}\left(Q_{N+1}=k \mid Q_{1}, \ldots Q_{N}\right)=1 /(N+1)
$$

Say that $I_{k}^{(N)}$ percolates if the point $\left(x_{k}, y_{k}, U_{k}\right)$ percolates. From Construction 2, we see that the point $\left(x_{N+1}, y_{N+1}, U_{N+1}\right)$ percolates if and only if $I_{Q_{N}}^{(N)}$ percolates and $\left(x_{N+1}, y_{N+1}, U_{N+1}\right)$ is green.

To put it formally, for every $k \in[1, N]$ let $V_{k}$ be the indicator function that $\left(x_{k}, y_{k}\right)$ is green and $W_{k}$ be the indicator function that $\left(x_{k}, y_{k}\right)$ percolates. Put also $W_{0}=1$. Then we have

$$
W_{N+1}=V_{N+1} W_{Q_{N}}
$$


Since the random variables $V_{n}$ are independent of the random variables $Q_{n}$, we can extend (4) by further conditioning on the random variables $V_{n}, W_{n}$ :

$$
\mathbb{P}\left(Q_{N+1}=k \mid Q_{1}, \ldots Q_{N}, V_{1}, \ldots V_{N}, W_{0}, \ldots W_{N},\right)=1 /(N+1)
$$

Using (6) together with (5), we can describe the law of the family of random variables $\left(W_{n}\right)$ as follows.

- First, $W_{0}=1$.

- Recursively at time $N \geq 1$,

- choose $Q_{N}$ uniformly at random on $[0, N]$, independently of the past.

- If $W_{Q_{N}}=0$, then $W_{N}=0$.

- If $W_{Q_{N}}=1$, then independently of the past, choose either $W_{N}=1$ or $W_{N}=0$ with respective probabilities $\alpha, 1-\alpha$.

Comparing with Section 3.1, we check that it is exactly the same mechanism as the urn scheme with initial condition $B_{0}=1-\alpha, W_{0}=\alpha$. So we can state

Proposition 1 Let $A_{n}$ be the number of percolating points in the set

$$
\left\{\left(x_{1}, y_{1}, U_{1}\right),\left(x_{2}, y_{2}, U_{2}\right), \ldots\left(x_{n}, y_{n}, U_{n}\right)\right\}
$$

Then the sequence $\left(A_{n}\right)$ has the same law as $\left(W_{n}\right)$, where $W_{n}$ is the ttotal weight of white balls in an urn scheme with replacement matrix

$$
\left(\begin{array}{cc}
1 & 0 \\
1-\alpha & \alpha
\end{array}\right)
$$

and initial condition $(1-\alpha, \alpha)$.

\subsection{The general case}

We use here the same assumptions on the function $\beta$ as in Theorem 1 and we keep the notation from Section 3.2.

Let $M$ be the number of points of the process $\mathcal{N}$ with $y$-coordinate greater that $h$. Then conditionally on $M$, the interval $[0,1]$ is cut into $M+1$ subintervals. We define percolating and non-percolating subintervals as in the previous subsection and denote by $W$ the number subintervals that percolate. Note that even if $M=0, W=1$ since by convention, we say that $\left(x_{0}, y_{0}\right)$ percolates.

Using the same arguments as in Section 3.2, we see that the family of lengths of these subintervals, which we can denote by $\left(l_{1}, \ldots l_{M+1}\right)$, is uniformly distributed on the simplex and that $\left(x_{M+1}, y_{M+1}, U_{M+1}\right)$ percolates if and only if it is green and $x_{M+1}$ lies in a subinterval which percolates.

Then adding $x_{M+1}$, we cut $[0,1]$ into $M+2$ subintervals and then we can see in which subintervals $x_{M+2}$ and whether the point $\left(x_{M+2}, y_{M+2}\right)$ percolates or not. Reasoning this way by induction, as in Section3.2, we see that conditionally on $M$ and $W$, we get an urn scheme with the same replacement matrix (1) but now the initial condition is $(M+1-W \alpha, W \alpha)$. 
Proposition 2 Let $A_{n}$ be the number of percolating points in the set

$$
\left\{\left(x_{1}, y_{1}\right),\left(x_{2}, y_{2}\right), \ldots\left(x_{M+n}, y_{M+n}\right)\right\}
$$

Then the sequence $\left(A_{n}\right)$ has the same law as $\left(W_{n}\right)$, where $W_{n}$ is the total weight of white balls in an urn scheme with replacement matrix

$$
\left(\begin{array}{cc}
1 & 0 \\
1-\alpha & \alpha
\end{array}\right)
$$

and random initial condition $(M+1-W \alpha, W \alpha)$.

It remains to study the joint law of $(M, W)$. First, the law of $M$ is Poisson with mean $\theta=(1 / h)-1$. Next, conditionally on $M$, using Section 2.3 , we get that $W$ has the same law as the number of points in $[0, M]$ in the regenerative set $R$ obtained from Construction 1 in Section 2.3.

This regenerative set $R$ is the trace of a random walk $\left(S_{n}\right)$ and the generating function of $S_{1}$ is the function $\psi$ given in Theorem 1 . Conditionally on $M$, we have

$$
\mathbb{P}(W=n+1 \mid M)=\mathbb{P}\left(Y_{n} \leq M, Y_{n+1}>M\right)=\mathbb{P}\left(Y_{n} \leq M\right)-\mathbb{P}\left(Y_{n+1} \leq M\right)
$$

For each $k$, we have

$$
\mathbb{P}\left(Y_{n}=k\right)=\left[t^{k}\right] \mathbb{E}\left(t^{Y_{n}}\right)=\left[t^{k}\right] \psi(t)^{n}
$$

where $\left[t^{k}\right] f(t)$ stands for the coefficient of the momomial $t^{k}$ in the function $f(t)$ viewed as a power series. By the theorem of residues,

$$
\left[t^{k}\right] \psi(t)^{n}=\frac{1}{2 i \pi} \int_{\mathcal{C}} \frac{\psi(t)^{n}}{t^{k+1}}
$$

Summing over $k$ in $(7)$ yields

$$
\mathbb{P}(W=n+1 \mid M)=\frac{1}{2 i \pi} \int_{\mathcal{C}} \frac{d t}{t} \psi(t)^{n}(1-\psi(t)) \frac{1-(1 / t)^{M+1}}{1-(1 / t)}
$$

and so finally,

$$
\mathbb{P}(M=m, W=n+1)=\frac{\theta^{m} e^{-\theta}}{m !} \frac{1}{2 i \pi} \int_{\mathcal{C}} \frac{d t}{t} \psi(t)^{n}(1-\psi(t)) \frac{1-(1 / t)^{m+1}}{1-(1 / t)}
$$

Comparing (8) with Theorem 1 and using Proposition 2, we can state:

Proposition 3 The sequence $\left(A_{n}\right)$ from Proposition 2 has the same law as the number of white balls in the urn process described in Theorem 1. 


\section{Proof of Theorem 1}

We assume in this section that the conditions of Theorem 1 are satisfied. We shall use the following property, see for instance [3] or [9] for a recent use of it:

Proposition 4 Suppose that a sequence of subordinators $S^{(n)}$ which are not compound Poisson converges in law to $S$. Then the law of the first passage time for $S^{(n)}$ converges in distribution to the law of the first passage time for $S$.

Let us go back to the construction of Section 2.2. If we only consider the points that percolate and that have a $y$-coordinate more than $1 / n$, this yields a regenerative set $R^{(n)}$ associated with the function $\alpha_{n}(x)=\alpha(x) \mathbf{1}_{\{x \geq 1 / n\}}$. According to Theorem $3, R^{(n)}$ is the range of a subordinator $S^{(n)}$ with exponent

$$
\phi^{(n)}(\lambda)=\exp \int_{0}^{1} \frac{(\lambda-1) \alpha(x)}{1+(\lambda-1) x} \mathbf{1}_{\{x \geq 1 / n\}} d x
$$

Thus $S^{(n)}$ converge to the subordinator $S$ with exponent given in Theorem 1 . Using Proposition 4 , we get the convergence

$$
T_{n}^{(\beta)} \stackrel{\operatorname{law}}{\rightarrow} T^{(\beta)}
$$

where $T_{n}^{(\beta)}$ stands for the first passage time for $S^{(n)}$

Since $S^{(n)}$ has a finite number of jumps inside a finite interval, it is a compound Poisson process. This means that the times between two consecutive jumps are iid, exponentially distributed random variables whose mean is given by

$$
m_{n}=\frac{1}{\phi^{(n)}(\infty)}=\exp \left(-\int_{1 / n}^{1} \frac{\alpha(x)}{x} d x\right) \sim c n^{-\alpha}
$$

for some constant $c>0$. Now let $K^{(n)}$ denote the number of of jumps of $S^{(n)}$ before exiting from $[0,1]$. Then conditionally on $K^{(n)}=k$, the first passage time $T_{n}^{(\beta)}$ has the same law as the sum of $k$ iid, exponentially distributed random variables with mean $m_{n}$ and variance $m_{n}^{2}$. Using the Chebyshev inequality, we get

$$
P\left(\left|T_{n}^{(\beta)}-K^{(n)} m_{n}\right|>A \mid K^{(n)}\right) \leq \frac{K^{(n)} m_{n}^{2}}{A^{2}}=\frac{K^{(n)} c^{2} n^{-2 \alpha}}{A^{2}}
$$

Next, remark that $K^{(n)}$ is the cardinal of the set $R^{(n)} \cap[0,1]$, that is, the number of percolating points with $y$-coordinate greater than $1 / n$. Using Proposition 3 , we get that $K^{(n)}$ has the same law as the total weight of white balls in the urn scheme described in Theorem 1:

$$
\alpha K^{(n)} \stackrel{\text { law }}{=} W_{L^{(n)}}
$$

where $L^{(n)}$ is the number of points of $\mathcal{N}$ with $y$-coordinate greater than $1 / n$. Note that $L^{(n)}$ is Poisson distributed with mean $n-1$ and therefore

$$
P\left(\left|L^{(n)}-n\right| \geq n^{2 / 3}\right) \rightarrow 0
$$


as $n \rightarrow \infty$. It follows from (10) that $n^{-\alpha} W_{L^{(n)}}$ and $n^{-\alpha} W_{n}$ have the same limit law, which is also the limit law of $n^{-\alpha} \alpha K^{(n)}$.

Taking $\delta>0$ and $A=n^{-\alpha / 4}$ in (9) yields

$$
P\left(\left|T_{n}^{(\beta)}-K^{(n)} m_{n}\right|>n^{-\alpha / 4}\right) \leq \mathbb{P}\left(K^{(n)}>\delta n^{\alpha}\right)+\frac{\delta}{n^{\alpha / 2}}
$$

This is true for every $\delta>0$ and we have seen that

$$
\mathbb{P}\left(K^{(n)}>\delta n^{\alpha}\right)
$$

has the same limit as

$$
\mathbb{P}\left(W_{n}>\delta \alpha n^{\alpha}\right)
$$

Since we know that the sequence $\left(n^{-\alpha} W_{n}\right)$ converges, it is tight and therefore, the upper bound in (11) goes to 0 as $n$ goes to infinity. So $T_{n}^{(\beta)}$ has the same limit law as $K^{(n)} m_{n}$, that is, the limit law described in Theorem 1. This concludes the proof.

Acknowledgments. This research was carried out while I was visiting Mexico during a thematic semester. I thank my mexican colleagues, in particular Geronimo Uribe, Maria Emila Caballero, Victor Rivero, Juan Carlos Pardo-Millan and Arno Siri-Jégousse for their warm welcome and interesting discussions on the topic. I thank the referee for a careful reading of the manuscript. And of course I would like to pay a tribute to Ron Doney, who has been interested in my work since I was a $\mathrm{PhD}$ student, invited me to Manchester, was a member of my habilitation jury and whose contributions to the study of Lévy processes are an inspiration for all probabilists working in the field.

\section{References}

[1] Alili, L.; Jedidi, W.; Rivero, V. On exponential functionals, harmonic potential measures and undershoots of subordinators. ALEA, Lat. Am. J. Probab. Math. Stat. 11, No. 2, 711-735 (2014).

[2] Bertoin, J. Subordinators: examples and applications. Lectures on probability theory and statistics (Saint-Flour, 1997), 1-91, Lecture Notes in Math., 1717, Springer, Berlin, 1999.

[3] Chaumont, L.; Doney, R. Invariance principles for local times at the maximum of random walks and Lévy processes. Ann. Probab. 38, No. 4, 1368-1389

[4] Deng, C.; Schilling, R. Complete Bernstein functions and subordinators with nested ranges. A note on a paper by P. Marchal. Electron. Commun. Probab. 21, Paper No. 78,5 p. (2016).

[5] Doney, R.; Rivero, V. Asymptotic behaviour of first passage time distributions for subordinators. Electron. J. Probab. 20, Paper No. 91, 28 p. (2015). 
[6] Janson, S. Limit theorems for triangular urn schemes. Probab. Theory Relat. Fields 134, No. 3, 417-452 (2006).

[7] Marchal, P. Nested regenerative sets and their associated fragmentation process. Mathematics and computer science. III, 461-470, Trends Math., Birkhäuser, Basel, 2004.

[8] Marchal, P. A class of special subordinators with nested ranges. Ann. Inst. Henri Poincar, Probab. Stat. 51, No. 2, 533-544 (2015).

[9] Mijatović, A; Uribe, G. Invariance principles for local times in regenerative settings. (2019) Preprint

[10] Pitman, J. Combinatorial stochastic processes. Ecole d'Eté de Probabilités de SaintFlour XXXII 2002. Lecture Notes in Mathematics 1875. Berlin: Springer

[11] Schilling, R.;Song, R.; Vondracek, Z. Bernstein functions. Theory and applications. de Gruyter Studies in Mathematics, 37. Walter de Gruyter, Berlin, 2010. 